\title{
Voice Handicap Index Emotional Subscale
}

National Cancer Institute

\section{Source}

National Cancer Institute. Voice Handicap Index Emotional Subscale. NCI Thesaurus. Code C128068.

A subscale of the Voice Handicap Index that measures an individual's perception of the emotional impact of their voice handicap. 